\title{
Puntas de proyectil líticas de la cuenca media e inferior del río Coyle (Santa Cruz): aportes al uso del espacio regional y a la distribución espacial del diseño Magallanes IV
}

\author{
Silvana Espinosa, Juan Bautista Belardi y Flavia Carballo Marina \\ Recibido 24 de julio 2020. Aceptado 01 de septiembre 2020
}

\begin{abstract}
RESUMEN
Se presenta el análisis tecnotipológico de 39 puntas de proyectil líticas registradas en la cuenca media e inferior del río Coyle. Se reconocen cinco subgrupos tipológicos manufacturados en rocas locales de alta disponibilidad. Las puntas fueron abandonadas con potencial de reactivación; predominan aquellas del sistema de armas arrojadizas, y 31 son semejantes al diseño Magallanes IV. La información complementa discusiones sobre el uso del espacio por parte de grupos cazadores del Holoceno tardío y suma la cuenca al análisis suprarregional de la distribución del diseño Magallanes IV. Los resultados son concluyentes con la propuesta que sostiene su circulación ininterrumpida desde el estrecho de Magallanes hasta el norte del río Santa Cruz.
\end{abstract}

Palabras clave: Puntas de proyectil; Magallanes IV; Río Coyle; Uso del espacio; Circulación.

\begin{abstract}
LITHIC PROJECTILE POINTS FROM THE MIDDLE AND LOWER BASIN OF THE COYLE RIVER, SANTA CRUZ: CONTRIBUTIONS TO THE STUDY OF REGIONAL SPATIAL USE AND THE SPATIAL DISTRIBUTION OF THE MAGALLANES IV STYLE. This paper introduces the technological analysis of 39 lithic projectile points from the middle and lower basin of the Coyle River. Five technological subgroups manufactured from local high availability raw material are identified. Points were abandoned with high resharpening potential, those from throwing weapon systems predominating, with 31 related to the Magallanes IV design. The data complements discussions on landscape use by Late Holocene hunter-gatherers and incorporates the Coyle River basin within the supraregional distribution of the Magallanes IV design. Results reinforce statements about the continuous spatial distribution of this point design from the Magellan Strait to north of the Santa Cruz River.
\end{abstract}

Keywords: Projectile points; Magallanes IV; Coyle River; Landscape use; Circulation.

\footnotetext{
Silvana Espinosa. Centro de Investigación y Transferencia (CIT) Santa Cruz, Consejo Nacional de Investigaciones Científicas y Técnicas (CONICET). Universidad Nacional de la Patagonia Austral (UNPA). Universidad Tecnológica Nacional (UTN), Lisandro de la Torre 860 (9400), Río Gallegos, Santa Cruz, Argentina. E-mail: silvanaespinosa@conicet.gov.ar

Juan Bautista Belardi. Instituto de Ciencias del Ambiente, Sustentabilidad y Recursos Naturales (ICASUR). Unidad Académica Río Gallegos (UARG). UNPA - Centro de Investigación y Transferencia (CIT) Santa Cruz, CONICET. Universidad Nacional de la Patagonia Austral (UNPA). Universidad Tecnológica Nacional (UTN). Campus universitario. Av. Gregores y Piloto Rivera s/n (9400), Río Gallegos, Santa Cruz, Argentina. E-mail: juanbautistabelardi@gmail.com

Flavia Carballo Marina. ICASUR, UARG, UNPA. Campus universitario Av. Gregores y Piloto Rivera s/n (9400), Río Gallegos, Santa Cruz, Argentina. E-mail: flaviacarballomarina@gmail.com
}

Intersecciones en Antropología 21(2), julio-diciembre: 201-211. 2020. ISSN-e 1850-373X

https://doi.org/10.37176/iea.21.2.2020.560

Facultad de Ciencias Sociales - UNICEN - Argentina 


\section{INTRODUCCIÓN}

El estudio de las puntas de proyectil líticas se ha empleado para conocer aspectos tecnológicos, buscar patrones temporales de variación morfológica y realizar seriaciones temporo-espaciales con significado cultural. En el sur de Patagonia, la cuenca del río Coyle (provincia de Santa Cruz) carecía de estudios sobre la diversidad y características tecnológicas de las puntas de proyectil líticas, sus diseños y su evaluación en función del uso del espacio regional y la circulación de información. Este último aspecto es relevante, ya que la cuenca se ubica entre dos regiones que cuentan con investigaciones al respecto, como el estrecho de Magallanes y el Campo Volcánico de Pali Aike (CVPA) (Gómez Otero, 1987; Nami, 1991; Charlin y González-José, 2012, entre muchos otros) al sur, y la cuenca del río Santa Cruz (Franco et al., 2010), al norte.

El objetivo de este trabajo es presentar la información de 44 piezas bifaciales líticas, 39 de las cuales son puntas de proyectil enteras y fragmentadas, procedentes de la cuenca media e inferior del río Coyle, analizar su distribución espacial y su adscripción al diseño de puntas de proyectil denominado Período IV (Bird, 1993) o Magallanes IV (sensu Gómez Otero, 1986-1987); finalmente, se busca integrar la cuenca a la discusión sobre la circulación regional de este diseño particular. Las piezas fueron recuperadas en superficie $y$ al aire libre $(n=28)$ como resultado de evaluaciones de impacto ambiental Ilevadas a cabo por este equipo de trabajo a lo largo de más de dos décadas; a ellas se suman otras 16 puntas de la colección Horst Thierauf, depositada en el Museo Regional Provincial "Padre Manuel Jesús Molina", de la ciudad de Río Gallegos, Santa Cruz. Se caracterizan los ejemplares según atributos técnicos y morfológicos y las trayectorias de vida útil a partir de índices de mantenimiento y de recambio de piezas.

\section{La cuenca del río Coyle: ambiente y arqueología}

La cuenca del río Coyle es una de las más extensas del sur de Santa Cruz (Figura 1). Su paisaje fue modelado por la acción glacifluvial y está compuesto por varios niveles de terrazas y mesetas sedimentarias planas de origen continental y marino. La escasa cobertura edáfica y vegetal facilita la erosión hídrica (Cáceres y Caballero, 2006). Es un ambiente de estepa graminosa xérica y arbustiva (Cuadra y Oliva, 1996). En su cuenca media e inferior no posee cuevas ni aleros debido a la escasa compactación del sedimento Terciario (Carballo Marina et al., 2000-2002); estos abrigos solo se encuentran en las coladas basálticas de las márgenes de los ríos Santa Cruz y Gallegos, que enmarcan a la cuenca del río Coyle por el norte y el sur respectivamente.

El predominio de la erosión genera condiciones de buena visibilidad arqueológica, lo cual permitió el análisis distribucional del registro arqueológico en las dos unidades de paisaje que caracterizan a la cuenca media e inferior del Coyle: Terrazas (con alta frecuencia de cuencas endorreicas de distribución

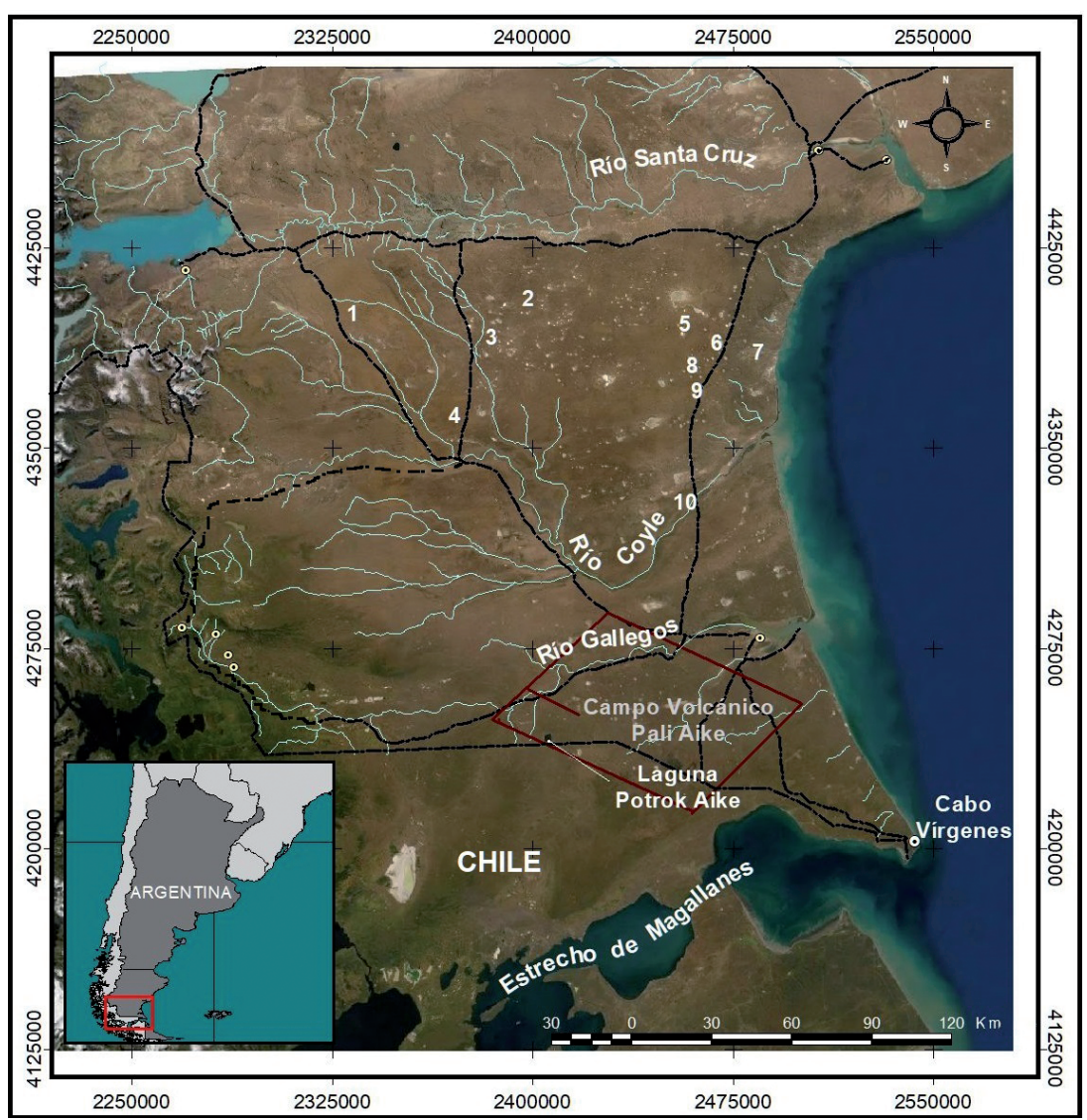

Figura 1. Sitios arqueológicos de donde provienen las puntas de proyectil. 1) El Cerrito, 2) Ea. La Tehuelche, 3) Cañadón Camusu Aike, 4) Campo Boleadoras, 5) Ea. La Maggie, 6) Laguna Los Luises, 7) Rincón del Buque, 8) Los Capones. Laguna 2, 9) Ea. Ototel Aike y 10) Cerro Redondo. 
homogénea y cañadones) y Fondo de valles (Belardi et al., 2006; Carballo Marina et al., 2011).

Hay alta disponibilidad y facilidad de acceso a rodados aptos para la talla en los paleocauces, laderas de las terrazas altas, cañadones, cuencas endorreicas y en el cauce actual del río, por lo cual la distribución de rocas es homogénea. Se encuentran principalmente dacitas, basaltos, andesitas y, en menor medida, madera silicificada, sílices y calcedonia (Espinosa et al., 2000). Las dimensiones de los guijarros de dacitas rondan los $300 \mathrm{~mm}$ en sus ejes mayores; hay otro grupo de rocas que se agrupan como Rocas de Grano Fino Oscuras (RGFO) (Charlin, 2005), que no superan los 95 mm.

La densidad artefactual regional es baja, con un orden de magnitud de $10^{-4}$, lo que apoya un uso humano poco intenso del espacio. La evidencia arqueológica en terrazas, cuencas endorreicas y cañadones de la unidad Terrazas indica que se habrían Ilevado a cabo los mismos tipos de actividades, vinculadas con el aprovisionamiento de rocas y actividades de talla, pero con diferencias en cuanto a su intensidad. Predominan los desechos de talla, núcleos y raederas, lo cual señala un importante componente expeditivo vinculado con la distribución homogénea y la alta disponibilidad y accesibilidad a las rocas. Las mayores densidades artefactuales se concentran en las cuencas endorreicas. Además, se destacan episodios de "talla al paso", que se asocian con una estrategia oportunista (Nelson, 1991). Los conjuntos arqueológicos de la unidad Fondo de valles son similares a los registrados en Terrazas, a los que se suman artefactos laminares sobre hojas y restos faunísticos, principalmente de guanaco (Lama guanicoe). Los artefactos de rocas alóctonas (obsidiana negra y gris verdosa veteada) y moluscos de procedencia atlántica, en baja frecuencia, habrían integrado circuitos de circulación de bienes e información relacionados con poblaciones localizadas principalmente al norte, al oeste y al este de la región. Las dataciones radiocarbónicas corresponden a ocupaciones del Holoceno tardío, desde 3200 años AP hasta momentos históricos (Carballo Marina et al., 20002002; 2011; Belardi et al., 2006). Sin embargo, fechados ca. 8800 años AP en cercanías de Fuentes del Coyle, en las nacientes del río (Borrero et al., 2006; Borrero y Borrazzo, 2011), permiten pensar en momentos más tempranos de ocupación en la región. En este sentido, el reciente hallazgo de una punta tipo "cola de pescado" (Matera et al., 2019) es coincidente con el uso temprano de la cuenca.

\section{LAS PUNTAS DE PROYECTIL DE DISEÑO MAGALLANES IV EN PATAGONIA MERIDIONAL}

Junius Bird estableció una secuencia arqueológica de cinco períodos culturales para Patagonia austral, basada sustancialmente, sobre el diseño de puntas de proyectil registradas en la cueva Fell, en el sector chileno del CVPA (Figura 1). El período más temprano, o Período I, datado entre 11.000 y 10.000 años AP, está asociado a puntas tipo "cola de pescado" manufacturadas sobre basalto. El Período II, conformado por variadas puntas de proyectil sobre hueso, se ubica alrededor de 9100 años AP. El artefacto diagnóstico del Período III son las puntas de proyectil triangulares apedunculadas y se desarrolló entre 8400 y 6560 años AP. En el Período IV, 3700 años AP, las puntas son de tamaño mediano, confeccionadas por adelgazamiento bifacial, de forma triangular, pedunculadas y con aletas. En el Período V, desde 650 años AP hasta momentos históricos, las puntas son pequeñas, delgadas, triangulares y con aletas, posiblemente utilizadas con arco y flecha (Bird, 1993).

Las investigaciones posteriores en Patagonia meridional, tanto en el estrecho de Magallanes y en el CVPA como en el río Santa Cruz, destacaron la distribución generalizada de las puntas de proyectil triangulares de tamaño pequeño con pedúnculo ancho y espeso asignables a Magallanes IV. Las dataciones más tempranas de estas puntas en el estrecho de Magallanes ubican al diseño entre 4560 años AP y 4280 años AP (Massone, 1989-1990) y 3475 años AP y 3725 años AP (Massone, 1979). Por su parte, Ratto (1994) analizó las puntas Magallanes IV y V, fechadas en los últimos 3400 años radiocarbónicos en el CVPA -cuencas de los ríos Chico y Gallegos- y logró establecer, al menos, tres sistemas técnicos de armas. En la cueva Don Ariel, en el río Chico, en distintos niveles estratigráficos se recuperaron puntas de proyectil pedunculadas con fechados posteriores a 4000 años AP y se relacionaron con el nivel Cultural tardío (Massone, 1989-1990), correspondiente a los Períodos IV y V de Bird (Nami, 1999).

Se realizó un estudio comparativo de puntas Magallanes IV de las colecciones privadas -Wood y Warne- provenientes de cercanías del estrecho de Magallanes y del centro-este de la provincia de Santa Cruz, respectivamente. Se analizaron los valores de los pedúnculos y limbos y la razón entre ellos. Los resultados indican un bajo nivel de 
integridad y un alto grado de reactivación de los ejemplares (Franco et al., 2005). La gran semejanza del diseño y el uso de materias primas locales no respondería a invenciones independientes o al intercambio en este extenso espacio, sino a la circulación de la información sobre un modelo exitoso (Franco et al., 2010).

Asimismo, en el CVPA, utilizando el modelo de asignación funcional propuesto por Ratto (1994), se exploraron las decisiones tecnológicas relacionadas con factores ecológicos y sociales que habrían afectado las estrategias de caza. Las piezas analizadas corresponden en un $76 \%$ a armas arrojadizas sin almacenamiento de energía, y en un 19\%, a arco y flecha (Banegas et al., 2014). Los cabezales son característicos del Período IV. Las diferencias entre los sistemas de armas radican en sus componentes; en las armas arrojadizas, el elemento de importancia es el ápice, mientras que las no arrojadizas conforman un sistema bimodular, compuesto por el limbo y el pedúnculo. Varias líneas de análisis observaron que la reducción afecta principalmente la forma y el limbo (Charlin y González-José, 2012; Banegas et al., 2014; Alberti y Cardillo, 2016).

\section{METODOLOGÍA}

El estudio de las puntas de la cuenca media e inferior del río Coyle siguió el análisis tecnomorfológico propuesto por Aschero (1975, 1983). Además, se consignó la ubicación geográfica de las puntas, la materia prima lítica y, finalmente, la conformación de subgrupos tipológicos y la asociación con el diseño Magallanes IV, de mayor expresión regional.

Los atributos analizados fueron la presencia de fracturas, las dimensiones de las piezas (longitud, ancho y espesor, tanto de la pieza como del pedúnculo), la sección, la serie técnica y la forma base. Se utilizó el ancho de la raíz del pedúnculo como un atributo diagnóstico para establecer el tipo de arma (Ratto, 1991, 1994).

Se aplicaron pruebas de estadística descriptiva y los Índices de Reavivamiento (Iriarte, 1995) -que mide el cociente entre el largo y el espesor máximos- y el de Descarte (Amick, 1996) -que lo hace a partir del cociente entre a) bases y pedúnculos + limbos y b) ápices + limbos-, con el fin de evaluar la variación morfológica y el proceso de mantenimiento de las piezas, respectivamente.

\section{PRESENTACIÓN Y ANÁLISIS DE LOS DATOS}

La muestra consta de 44 piezas bifaciales: 39 puntas de proyectil pedunculadas enteras y fracturadas manufacturadas en diversas materias primas, un ápice de basalto, una punta reciclada como cuchillo de basalto, dos preformas de punta (una fracturada de basalto y otra entera de dacita) y un fragmento de biface de basalto.

Las puntas de proyectil se registraron, tanto en concentraciones como en hallazgos aislados en Terrazas, a partir de la implementación de transectas lineales en el marco de trabajos distribucionales. Se carece de información acerca de la metodología de recolección de las puntas de proyectil de la colección Thierauf. El grado de integridad de la muestra es alto, ya que el 93,02\% de los ejemplares conserva más del 75\% de sus elementos (ápice, limbo, pedúnculo, etc.). Asimismo, la abrasión (Borrazzo, $2006)$ es leve (30\%). De esta manera, resulta confiable para el análisis y permite la asignación adecuada de los subgrupos tipológicos (Tabla 1 y Tabla 1 Suplementaria).

\section{Materias primas y subgrupos tipológicos}

Es preeminente el uso del basalto de diferentes variedades $(79,5 \% ; n=31)$ en la manufactura de

\begin{tabular}{|l|c|c|c|c|c|}
\hline \multicolumn{1}{|c|}{$\begin{array}{c}\text { Subgrupos } \\
\text { tipológicos }\end{array}$} & Basalto & Dacita & $\begin{array}{c}\text { Madera } \\
\text { silicificada }\end{array}$ & RGFO & TOTAL \\
\hline $\begin{array}{l}\text { Pedúnculo destacado y } \\
\text { hombros }\end{array}$ & 1 & 0 & 0 & 0 & 1 \\
\hline $\begin{array}{l}\text { Pedúnculo diferenciado } \\
\text { y hombros }\end{array}$ & 21 & 1 & 1 & 3 & 26 \\
\hline $\begin{array}{l}\text { Pedúnculo diferenciado } \\
\text { y aletas entrantes }\end{array}$ & 4 & 3 & 0 & 0 & 7 \\
\hline $\begin{array}{l}\text { Pedúnculo esbozado y } \\
\text { aletas en espolón }\end{array}$ & 1 & 0 & 0 & 0 & 1 \\
\hline $\begin{array}{l}\text { Pedúnculo esbozado sin } \\
\text { aletas }\end{array}$ & 1 & 0 & 0 & 0 & 1 \\
\hline Indiferenciadas & 3 & 0 & 0 & 0 & 3 \\
\hline TOTAL & 31 & 4 & 1 & 3 & 39 \\
\hline
\end{tabular}

Tabla 1. Subgrupos tipológicos y frecuencias de puntas de proyectil por materia prima. 
los diversos subgrupos tipológicos. También los hay confeccionados con dacita, madera silicificada y RGFO. Como se mencionó, todas las rocas están disponibles en el área de estudio en rodados de entre 100 y $300 \mathrm{~mm}$.

Se reconocieron cinco subgrupos tipológicos pedunculados (Tabla 1, Figura 2). Las bases de los pedúnculos son predominantemente concavilíneas (Aschero, 1983). Los subgrupos de pedúnculo diferenciado contienen al $84,6 \%$ de las piezas, lo que indica la prevalencia de una morfología particular.

\section{Fracturas}

A pesar del alto grado de integridad del conjunto, el $75 \%(n=33)$ de las piezas presenta fractura: el $33,3 \%(n=11)$ en el ápice, el $27,27 \%(n=9)$ en el ápice y en alguna de las aletas, el 18,18\% $(n=6)$ en el pedúnculo, el $12,12 \%(n=4)$ en las aletas y el $6,06 \%(n=2)$ restante en el limbo.

Todas las puntas de proyectil son piezas terminadas; por lo cual las fracturas no corresponden a errores de la manufactura. Por el contrario, están asociadas con el impacto o con acciones posdepositacionales. Respecto de su posición, el 48,4\% $(n=16)$ son transversales, el 36,36\% $(n=12)$, oblicuas, y el $15,15 \%(n=5)$, longitudinales.

\section{Tamaños}

Se contabilizan los ejemplares completos y con fracturas que no alteren las tendencias de las dimensiones longitud, anchura y espesor ( $n=19$, $48,71 \%$ ). Lo mismo se realizó con los pedúnculos, ya que el área de enmangue tiende a estandarizarse; $\leq 10 \mathrm{~mm}$ se asigna a arco y flecha, $y \geq$ a $10 \mathrm{~mm}$ se asigna como arma arrojadiza y/o de mano (lanza o dardo) (Ratto, 1991).

Las puntas de basalto son las que presentan mayor frecuencia de ejemplares enteros. Su longitud tiene una distribución relativamente homogénea, dado que el $60 \%$ mide entre 35 y 47 mm, con valores extremos de 34,6 mm y 60,9 mm (Figura 3 A). El ancho se muestra aún más homogéneo; el $70 \%$ se presenta entre 20 y $30 \mathrm{~mm}$, y los valores extremos son $16 \mathrm{~mm}$ y $39 \mathrm{~mm}$ (Figura 3 B). Por último, el espesor concentra un $60 \%$ de la muestra entre 6,7 y 7,7 mm, con valores extremos de 4,6 mm y 8,1 $\mathrm{mm}$ (Figura $3 \mathrm{C}$ ). Las puntas de dacita y madera silicificada son levemente más largas, mientras que el ancho se ubica en los valores promedio (Figuras 3 A, B y C) y pertenecen al subgrupo tipológico pedúnculo diferenciado y hombros, y las más pequeñas, al subgrupo tipológico pedúnculo diferenciado y aletas entrantes. Se observaron diferencias importantes entre los espesores de los limbos y de la raíz de los pedúnculos en siete ejemplares, dos de dacita y cuatro de basalto de pedúnculo diferenciado y aletas, y una en RGFO del subgrupo pedúnculo diferenciado y hombros.

\section{Pedúnculo}

El tamaño del pedúnculo pudo medirse en un número mayor de piezas $(\mathrm{N}=35,89,7 \%)$. En la longitud predomina el intervalo $10-19,9 \mathrm{~mm}$ en el $56,28 \%(\mathrm{n}=22)$ de las puntas. La medida más frecuente es 9,9 mm (21,05\%) en el intervalo 0-9,9 $\mathrm{mm}$, que alcanza $40 \%(\mathrm{n}=14)$. La media es 11,85

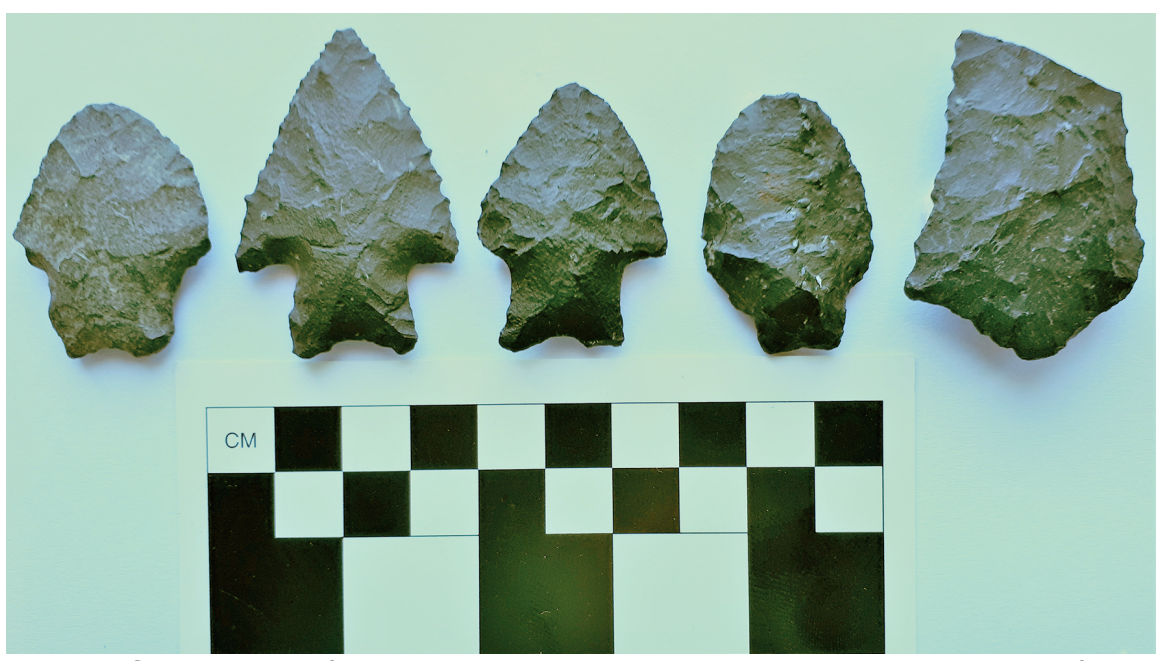

Figura 2. Subgrupos tipológicos de puntas de proyectil, de izquierda a derecha: A) pedúnculo destacado y hombros; B) pedúnculo diferenciado y aletas entrantes; C) pedúnculo diferenciado y hombros; D) pedúnculo esbozado sin aletas; y E) pedúnculo esbozado en espolón. $\mathrm{mm}$.

El ancho de los pedúnculos tiene valores superiores a la longitud. Los de mayor longitud se ubican entre $6,6 \mathrm{~mm}$ y $22 \mathrm{~mm}(\mathrm{n}=16 ; 42,1 \%)$. La media es $16,1 \mathrm{~mm}$. Son valores asociados con lanzas y/o armas de mano y tres casos asignables a flecha. El espesor del $36,8 \%$ del conjunto recae entre los valores 4,5 y $5,5 \mathrm{~mm}$. El $42,10 \%$ lo hace entre 7 y $8 \mathrm{~mm}$. La 


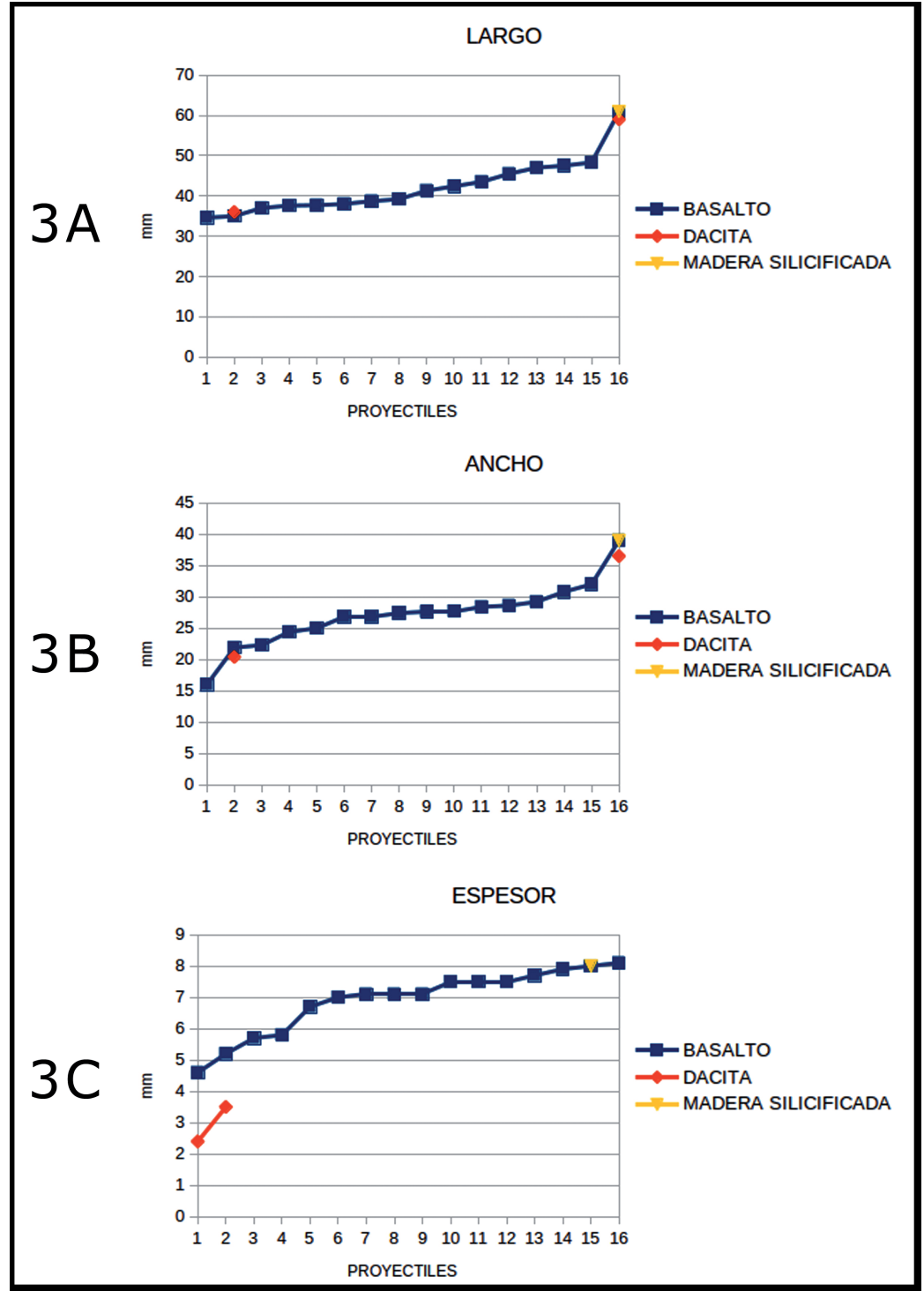

Figura 3. Dimensiones de las puntas de proyectil; A) largo; B) ancho; C) espesor.
Los bordes pudieron ser observados en 16 piezas, $14(87,5 \%)$ de ellas resultaron de forma simétrica, y el $12,5 \%$ son asimétricos.

\section{Formas base}

Se determinaron en todas las puntas. El $84,61 \%(n=33)$ son bifaces, y el 15,38\% $(n=6)$ son lascas. Predomina el uso del adelgazamiento bifacial. La muestra contiene una muy baja frecuencia de preformas; dos bifaces en estadio 2 (Nami, 1986).

\section{Serie técnica}

En los 39 ejemplares, la manufactura se realizó por presión. Predominan los retoques cortos paralelos regulares e irregulares $(79 \%)$, a los que se suman algunos retoques laminares. Se observan negativos de tamaño retalla en un 52,38\% de los casos. El $26 \%$ de la muestra combina ambas series técnicas. Los microrretoques, presentes en el 26\% de los casos, son muy frecuentes en la formatización de las aletas (81\%) y en los bordes del limbo. En este último, se registraron lasmedia del espesor es 5,86 mm, con tendencia a ser espesos (Franco et al., 2010).

\section{Sección}

Los ejemplares analizados presentan una distribución bimodal, el 52,27\% ( $\mathrm{n}=23)$ tiene sección biconvexa y el $43,18 \%(n=19)$, planoconvexa. La primera ofrece una trayectoria de vuelo estable y la segunda la vuelve inestable (Ratto, 2003).

El subgrupo más frecuente, de pedúnculo diferenciado y hombros, se inclina hacia la sección biconvexa ( $n=17,65,38 \%)$, y el $34,61 \%(n=9)$, a la planoconvexa. El subgrupo pedúnculo diferenciado y aletas presenta secciones biconvexas $(\mathrm{n}=3)$ y planoconvexas $(\mathrm{n}=3)$ en la misma proporción. cados paralelos transversales y diagonales. Las bases de los pedúnculos presentan adelgazamiento por retoque, que combina lascados paralelos cortos y laminares. En la raíz del pedúnculo de 14 ejemplares se observaron retoques con terminación quebrada y retoques marginales que interrumpen la continuidad de la sección, relacionados con el rejuvenecimiento de los bordes por adelgazamiento.

\section{Índices de Reavivamiento y Descarte}

El Índice de Reavivamiento (Iriarte, 1995) (Figura 4) muestra valores bajos y homogéneos; la correlación con la longitud de las piezas es negativa y media $(-0,47)$. Por su parte, el Índice de Descarte (Amick, 1996) señala la preponderancia de las bases y pedúnculos + limbos $(n=36)$ sobre un ápice, mayoritariamente en la superficie de las terrazas. 


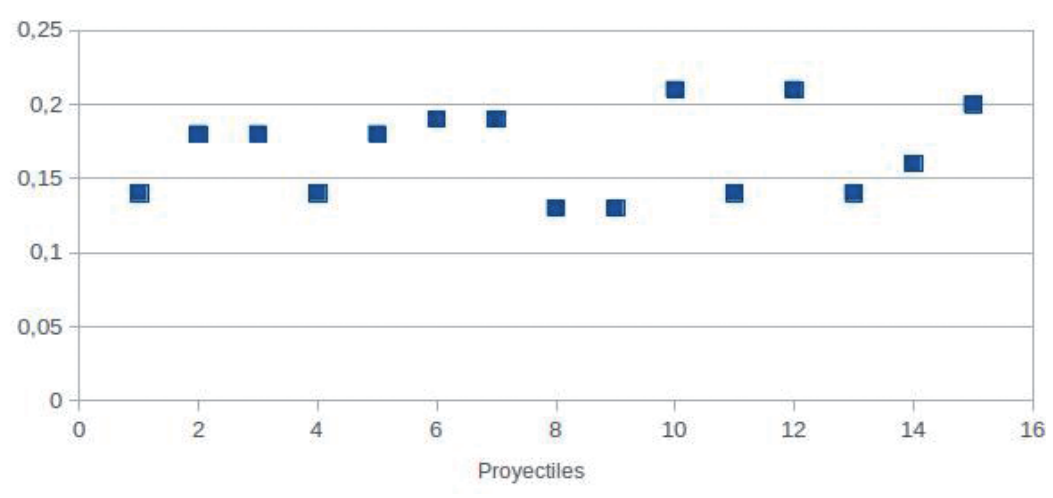

Figura 4. Índice de Reavivamiento (Iriarte, 1995).

\section{DISCUSIÓN Y CONCLUSIONES}

La muestra de puntas de proyectil de la cuenca media e inferior del río Coyle presenta una integridad alta $(93,02 \%)$, a pesar de provenir de contextos de superficie. Un nivel de integridad menor (65,9\%) se observó en CVPA (Banegas et al., 2014), donde los hallazgos corresponden a contextos de superficie y estratigrafía. Esta tendencia podría deberse, en parte, a la necesidad de prolongar la vida útil de las puntas, dada la distribución espacial diferencial de rocas aptas para la talla que ofrece el CVPA (Charlin y Pallo, 2013). Por el contrario, en la cuenca del río Coyle, la distribución homogénea y el fácil acceso a las rocas (Espinosa et al., 2000) habría facilitado el descarte y reemplazo de las puntas, aun de aquellas con potencial de reactivación. Además, la mayor cobertura vegetal de esta cuenca podría haber amortiguado la caída de los tiros errados.

Las materias primas utilizadas en la manufactura de las puntas son basaltos, RGFO, dacita gris y madera silicificada, todas disponibles localmente (Belardi et al., 2006). Las puntas de basalto son más frecuentes en la cuenca media, mientras que aquellas de rocas silíceas se distribuyen en frecuencias levemente mayores en la cuenca inferior. Esto estaría asociado con la oferta litológica de cada espacio, ya que los subgrupos tipológicos no muestran una selección por materia prima. Se destaca la mayor inversión de energía en la manufactura de artefactos bifaciales de dacita gris, de excelente calidad para la talla, de modo similar a lo observado en la margen sur del río Santa Cruz (Franco, 2008). Por su parte, en el CVPA, la mayoría de las piezas se confeccionaron en RGFO volcánicas (Nami, 1999; Charlin y González-José, 2012; Charlin y Pallo, 2013; Banegas et al., 2014).

Las formas base mayoritarias son bifaces y la formatización se logró por adelgazamiento bifacial
(Hocsman y Escola, 2006-2007), empleando presión para reducir el espesor sin afectar el ancho. Esta característica también se registró en las puntas de proyectil del CVPA y de Cabo Vírgenes (Franco et al., 2009). Los lascados son paralelos transversales y diagonales $y$, en algunos casos, cortos. Se obtuvieron productos relativamente estandarizados, biconvexos y planoconvexos. La baja frecuencia de preformas y el predominio de productos finales con series técnicas de baja inversión de energía indicarían la aplicación de una técnica específica en pocas etapas (Nami, 1986). El $52,38 \%$ de las puntas posee negativos de retalla y en el $81 \%$ el retoque está presente solo en la regularización de las aletas. Todo ello concuerda con el componente expeditivo señalado para la tecnología lítica en la unidad Terrazas (Belardi et al., 2006). De esta manera, los subgrupos tipológicos (Tabla 1) reflejan variaciones del diseño compuesto por las puntas triangulares con pedúnculo. Esta tendencia difiere con la mayor variabilidad de formas finales que logra la reducción bifacial como técnica de rejuvenecimiento de puntas, tal lo observado en el norte de Patagonia (Alberti y Cardillo, 2016).

En referencia a los subgrupos tipológicos (Figura 2 ), el de puntas triangulares con pedúnculo diferenciado y hombros (Aschero, 1983) es el más frecuente; están manufacturadas principalmente sobre basalto y tienen amplia distribución espacial (Tabla 1). El $62 \%$ presenta el ápice fracturado y hay un solo caso de fractura en la raíz del pedúnculo.

El subgrupo tipológico pedúnculo diferenciado $y$ aletas entrantes $(n=6)$, con ejemplares de dacita y de basalto, tiene una distribución semejante al primero. Las fracturas se registran en todos los elementos de las puntas de dacita $(n=3)$, que tienden a ser de tamaño más pequeño. Dentro de este subgrupo, se destaca un ejemplar que posee bordes dentados o denticulados (Aschero, 1975, 1983); está entero y podría tratarse de una pieza con poco uso, ya que el Índice de Reavivamiento es bajo (ver Figuras 2 B y 4). El borde dentado o denticulado también se ha observado en un biface o preforma con la base fracturada. Ambas piezas son de basalto. Este diseño se aprecia en puntas de proyectil de los Períodos III y IV (Bird, 1993: Figuras 14 y 15) y también ha sido reconocido en Tapi Aike (Matera et al., 2019: Figura 6) y en dos 
casos en el río Penitente, en las nacientes del río Gallegos (observaciones personales). El aserrado ha sido relacionado con connotaciones identitarias o simbólicas, aun en contextos bélicos, ya que análisis experimentales muestran que la penetración de las puntas de proyectil no se ve significativamente mejorada (Loendorf et al., 2015). Por otra parte, estudios combinados de cladística y morfometría geométrica indican la convergencia de un mismo diseño aserrado en distintos momentos bajo condiciones sociales y ambientales diferentes. El borde aserrado se habría usado para desgarrar las heridas provocadas tanto a presas como a enemigos (Smallwood et al., 2018). En el sur de Patagonia, el diseño de puntas aserradas se manifiesta en baja frecuencia. Por lo tanto, si el aserrado mejorara su desempeño, los proyectiles debieran encontrarse más representados. No obstante, es un diseño versátil (Nelson, 1991), que podría haberse utilizado tanto como arma de caza o como cuchillo en el procesamiento de presas. Por otra parte, si se tratara de una característica identitaria, su baja frecuencia reflejaría su expresión a nivel individual.

El subgrupo pedúnculo esbozado y aletas en espolón está integrado por un único caso manufacturado por presión en RGFO. Sus dimensiones coinciden con los intervalos más representados. Al menos un ejemplar similar ha sido registrado en la laguna Tom Gould (CVPA) (Nami, 1984, Figura 2E), manufacturado por adelgazamiento bifacial, acabado por presión y asignado al Período III, y otro en la cuenca del lago Cardiel (Goñi et al., 2005), a $250 \mathrm{~km}$ al NO del río Coyle. Es un diseño poco frecuente y en todos los casos manufacturado en rocas locales (incluido el canal de Beagle, Orquera comunicación personal en Goñi et al., 2005). Esta distribución espacial pone de relieve la extendida circulación de diseños particulares y la conectividad de la cuenca del río Coyle con otras regiones.

Finalmente, el subgrupo de pedúnculo destacado y hombros $(\mathrm{n}=1)$, confeccionado sobre basalto, presenta microrretoques en los bordes, vinculados con tareas de reactivación.

La posición de las fracturas y las huellas sobre las caras y bordes, los lascados con terminaciones quebradas, las fracturas transversales en los limbos y los negativos discontinuos sobre los bordes son interpretados como evidencias de impacto (Escola, 2014). Las fracturas rectas (Weitzel, 2011) sobre las caras podrían responder a causas posdepositacionales. La ausencia de fracturas en la raíz del pedúnculo estaría relacionada con la textura de las rocas locales, que debió favorecer la adherencia de los pedúnculos al astil, en detrimento de rocas vítreas (Loendorf et al., 2015). En el CVPA, las fracturas en el ápice son más frecuentes en las flechas; a su vez, en las lanzas hay una mayor ocurrencia de fracturas múltiples y en las aletas, como consecuencia, en parte, de la inestabilidad del arma (Banegas et al., 2014).

En relación con la vida útil de los ejemplares, el $33 \%$ del conjunto presenta ápices muy adelgazados respecto del limbo, diferencias entre los espesores de los limbos y de los pedúnculos y terminaciones quebradas de los lascados sobre las caras. Esto refleja el mantenimiento del proyectil enmangado. Sin embargo, el Índice de Reavivamiento (Iriarte, 1995) (Figura 4) indica el reacondicionamiento de las puntas sin que se redujera significativamente la longitud. Es decir, el adelgazamiento bifacial no modificó su forma original, sino que la estrategia tecnológica fue mantener relativamente constante el ancho de la pieza. Refuerza esta tendencia que solo un ejemplar presenta negativos paralelos profundos y ha sido reciclado en un cuchillo.

El Índice de Descarte (Amick, 1996) señala una estrategia de recambio de puntas aún sin estar agotadas y/o con potencial para ser recicladas, tal como lo indica la preponderancia de las bases y pedúnculos + limbos. Esto, registrado tanto en sitios como en forma aislada en las terrazas, podría haber ocurrido durante las tareas de obtención y procesamiento de las presas. Un patrón similar de abandono (Kuhn, 1995) se observa en raederas enteras y/o de dimensiones suficientes para ser reutilizadas. En este caso, el abandono de puntas estaría relacionado tanto con la alta disponibilidad de rocas ya mencionada como con el equipamiento de cazadores (Kuhn, 1995), los que circulan por espacios abiertos con limitadas posibilidades de ocultamiento. Por otro lado, el retorno a los campamentos donde reacondicionar las armas debió ser habitual (Andrefsky, 2008), como ejemplifican los conjuntos artefactuales localizados en las cuencas endorreicas -que son aquellos de mayor densidad y riqueza. Dichas geoformas, distribuidas de manera homogénea y con oferta de agua, fauna y rocas, pudieron constituir un circuito previsible que articulara las actividades de caza y la movilidad residencial (Carballo Marina et al., 2000-2002).

En todo el conjunto se reconocen dos sistemas técnicos de armas (Ratto, 1991, 1994); predomina 
el de las armas arrojadizas sin almacenamiento de energía $(n=34)$, integrado por los subgrupos tipológicos pedúnculo diferenciado y hombros, pedúnculo diferenciado y aletas entrantes, y pedúnculo destacado y hombros. La superficie de refuerzo se orienta a evaluar el esfuerzo mecánico que soporta el proyectil; refleja la relación entre el espesor máximo y el ancho del cabezal en ese sector (Ratto, 2003). Los valores obtenidos para la muestra del Coyle son bajos a medios, en el rango de 0,17 a 0,32, siendo la moda 0,23, 0,25 y 0,28 . Las piezas más angostas y pequeñas presentan valores bajos de superficie de refuerzo, posiblemente relacionados con arco y flecha, dos de ellos del subgrupo tipológico pedúnculo diferenciado y aletas. Estos valores son semejantes a los descriptos para las armas arrojadizas de CVPA; en su mayoría, lanzas (Banegas et al., 2014: Tabla 3).

El $79,48 \%(n=31)$ de las puntas de proyectil bajo análisis son medianas, bifaciales, triangulares con pedúnculo y, morfológicamente, se engloban en el diseño Magallanes IV (Bird, 1938; Gómez Otero, 1986-1987) (Tabla 1). A su vez, las dimensiones y formas de los pedúnculos muestran similitud con el diseño Magallanes IV espesas de la colección Wood (Franco et al., 2005), aunque más cortos y delgados. El 48,5\% de los casos $(n=17)$ tiene un espesor superior a la media $(5,68 \mathrm{~mm})$ y comparten los mismos valores respecto del ancho, por lo cual todas serían asignadas a lanzas. La colección Warne tiene medidas que se diferencian de las otras dos, siendo las puntas más largas, angostas y delgadas (Franco et al., 2005) (Tabla 2). Por su parte, en el CVPA, las modificaciones en los tamaños y formas de lanzas y otras armas arrojadizas se deben al reciclado (Banegas et al., 2014: Tabla 9).

Las bases de los pedúnculos en la cuenca del río Santa Cruz poseen variaciones significativas solo en las formas cóncavas y rectas (Franco et al., 2009). Los autores observan que los pedúnculos más largos son más anchos y espesos en el sur (CVPA y Cabo Vírgenes) que en el norte del río Santa Cruz, lo cual sostiene la tendencia de la colección Wood. Las bases de los pedúnculos de las puntas de proyectil de la cuenca del Coyle son concavilíneas, y las medias de longitud y espesor poseen valores intermedios entre los conjuntos del CVPA y Cabo Vírgenes y los del norte del río Santa Cruz. Sin embargo, son más semejantes a los que se atribuyeron como lanzas en el CVPA (Banegas et al., 2014). En resumen, se observan similitudes con las puntas de proyectil de diseño Magallanes IV espesas registradas tanto al

\begin{tabular}{|l|l|l|l|}
\hline Proveniencia & Largo & Ancho & Espesor \\
\hline $\begin{array}{l}\text { Cuenca media e inferior } \\
\text { del río Coyle }\end{array}$ & 11,85 & 16,10 & 5,86 \\
\hline Colección Wood & 12,74 & 16,12 & 6,44 \\
\hline Colección Warne & 12,92 & 14,13 & 5,06 \\
\hline
\end{tabular}

Tabla 2. Medias de las dimensiones de los pedúnculos en $\mathrm{mm}$, Wood y Warne. Elaboración propia a partir de datos tomados de Franco et al. (2005, pp. 86-87).

sur como al norte de la cuenca del río Coyle. En su mayoría $(69,23 \%)$, pudieron integrar sistemas de armas arrojadizas, probablemente lanzas $y$, en el caso de los ejemplares más pequeños $(7,69 \%)$, el de arco y flecha. Se trata de dos sistemas que muy probablemente hayan coexistido.

Los resultados del análisis de puntas de proyectil aportan a la discusión del uso cazador recolector de la cuenca media e inferior del río Coyle. Configuran un diseño particular, de manufactura estandarizada lograda con baja inversión de energía, que se vio facilitada por la amplia disponibilidad de materia prima local. A la vez, insertan a la región en la discusión de la circulación de distintos diseños de puntas de proyectil y, en especial, del Magallanes IV espesas. En este sentido, confirma su distribución ininterrumpida desde el estrecho de Magallanes hasta el norte del río Santa Cruz, aun cuando se reconoce la variabilidad inherente a cada región. Esto se vincularía con la circulación de información, que empleó formas similares de transmisión de los modos de manufactura y/o técnicas de caza (Banegas et al., 2014; Cardillo y Charlin, 2014), y mostraría la conectividad entre los distintos espacios de Patagonia meridional.

\section{Agradecimientos}

Al personal de las estancias donde realizamos los trabajos de campo. A los tres evaluadores por sus valiosos aportes. A Luis Borrero por su generosa lectura del manuscrito.

\section{REFERENCIAS CITADAS}

Alberti, J. y Cardillo, M. (2016). Análisis morfométrico y tecnológico de artefactos bifaciales. Un caso de estudio del Golfo San Matías (Provincia de Río Negro, Argentina). Chungara, 48(1), 39-52. 10.4067/ S0717-73562016005000006

Amick, D. (1996). Regional patterns of Folsom mobility and land use in the American Southwest. World Archaeology, 27(3), 411-426. 
Andrefsky, W., Jr. (2008). Projectile point provisioning strategies and human land use. En W. Andrefsky J. (ed.), Lithic Technology: Measures of production, use, and curation (pp. 195-215). Cambridge University Press.

Aschero, C. (1975). Ensayo para una clasificación morfológica de los instrumentos líticos aplicada a estudios tipológicos comparativos. Informe al CONICET. MS.

Aschero, C. (1983). Ensayo para una clasificación morfológica de los instrumentos líticos aplicada a estudios tipológicos comparativos. Apéndices A-C revisión. Cátedra de Ergología y Tecnología, Facultad de Filosofía y Letras, Universidad de Buenos Aires, Buenos Aires. MS.

Banegas, A., Gómez Otero, J., Goye, S. y Ratto, N. (2014). Cabezales líticos del Holoceno tardío en Patagonia meridional: Diseños y asignación funcional. Magallania, 42(2), 155-174.

Belardi, J. B., Carballo Marina, F. y Espinosa, S. (eds.) (2006). Cazadores recolectores en la cuenca media e inferior del río Coyle. En La cuenca del río Coyle. Estado actual de las investigaciones (pp. 97-128). Universidad Nacional de la Patagonia Austral.

Bird, J. (1938). Antiquity and migrations of the early inhabitants of Patagonia. The Geographical Review, 28, 250-275.

Bird, J. (1993). Viajes y arqueología en Chile Austral, Chile. Ediciones de la Universidad de Magallanes.

Borrazzo, K. (2006). Tafonomía lítica en dunas: una propuesta para el análisis de los artefactos líticos. Intersecciones en Antropología, 7, 247-261.

Borrero, L. A. y K. Borrazzo (comps.) (2011). La geografía cultural del sudoeste de Patagonia Continental. En Bosques, Montañas y cazadores: investigaciones arqueológicas en Patagonia Meridional (pp. 7-36). CONICET; IMHICIHU.

Borrero, L., Franco, V., Martin, F., Barberena, R., Guichon, R., Belardi, J. B. y L'Heureux, L. (2006). Las cabeceras del Coyle: información arqueológica y circulación de poblaciones humanas. En J. B. Belardi, F. Carballo Marina y S. Espinosa (eds.), La cuenca del río Coyle. Estado actual de las investigaciones (pp. 75-95). Universidad Nacional de la Patagonia Austral.

Cáceres, A. y J. Caballero. (2006). Consideraciones generales sobre la cuenca del río Coyle o Coig. En J. B. Belardi, F. Carballo Marina y S. Espinosa (eds.), La cuenca del río Coyle. Estado actual de las investigaciones (pp. 21-28). Universidad Nacional de la Patagonia Austral.

Carballo Marina, F., Belardi, J. B., Espinosa, S. y Ercolano, B. (2000-2002). Tecnología y movilidad en el río
Coyle, Santa Cruz. Cuadernos del Instituto Nacional de Antropología y Pensamiento Latinoamericano, 19, 89-107.

Carballo Marina, F., Belardi, J. B. y Sáenz, J. L. (2011). Distribución espacial del registro arqueológico en la unidad de paisaje terrazas, cuenca media del río Coyle (provincia de Santa Cruz, Argentina). Magallania, 39(2), 207-222.

Cardillo, M. y Charlin, J. (2014). Morphological Diversification of Stemmed Projectile Points of Patagonia (Southernmost South America). Assessing Spatial Patterns by Means of Phylogenies and Comparative Methods. En R. Iovita y K. Sano (eds.), Multidisciplinary Approaches to the Study of Stone Age Weaponry, Vertebrate Paleobiology and Paleoanthropology. Springer. 10.1007/978-94-017-7602-8_18

Charlin, J. (2005). Utilización de materias primas líticas en el Campo Volcánico Pali Aike (Pcia. Santa Cruz, Argentina). Werken, 7, 39-56.

Charlin, J. y González-José, R. (2012). Size and shape variation in Late Holocene projectile points of Southern Patagonia: a geometric morphometric study. American Antiquity, 77(2), 221-242.

Charlin, J. y Pallo, M. (2013). Disponibilidad de materias primas líticas y uso del espacio en el interfluvio Gallegos-Chico (Pali Aike, Santa Cruz, Argentina). En A. F. Zangrando, R. Barberena, A. Gil, G. Neme, M. Giardina, L. Luna,...A. Tívoli (comps.), Tendencias teórico-metodológicas y casos de estudio en la arqueología de la Patagonia (pp. 307-316). Museo de Ciencias Naturales de San Rafael; Instituto Nacional de Antropología y Pensamiento Latinoamericano; Sociedad Argentina de Antropología.

Cuadra, D. y Oliva, G. (1996). Ambientes naturales de la provincia de Santa Cruz. Espacios, 6, 22-27.

Escola, P. (2014). Proyectiles líticos en contexto en Arroyo Seco 2: algo más que una tecnología para la caza. En G. G. Politis, M. A. Gutiérrez y C. Scabuzzzo (eds.), Estado actual de las investigaciones en el sitio arqueológico Arroyo Seco 2 (partido de Tres Arroyos, provincia de Buenos Aires, Argentina) (pp. 313-327). Serie Monográfica Número 5. Instituto de Investigaciones Arqueológicas y Paleontológicas del Cuaternario Pampeano-Consejo Nacional de Investigaciones Científicas y Técnicas-Universidad Nacional del Centro de la Provincia de Buenos Aires.

Espinosa, S., Belardi, J. B. y Carballo Marina, F. (2000). Fuentes de aprovisionamiento de materias primas líticas en los sectores medio e inferior del interfluvio CoyleGallegos (Pcia. de Santa Cruz). En Desde el país de los gigantes. Perspectivas arqueológicas en Patagonia (pp. 5-17). Universidad Nacional de la Patagonia Austral. 
Franco, N. (2008). La estructura tecnológica regional y la comprensión de la movilidad humana: tendencias para la cuenca del río Santa Cruz. En L. A. Borrero y N. V. Franco (comps.), Arqueología del extremo sur del continente americano. Resultados de nuevos proyectos (pp. 119-154). Consejo Nacional de Investigaciones Científicas y Técnicas-Instituto Multidisciplinario de Historia y Ciencias Humanas.

Franco, N., Cardillo, M. y Borrero, L. (2005). Una primera aproximación a la variabilidad presente en las puntas denominadas "Bird IV". Werken, 6, 81-95.

Franco, N., Castro, A., Cardillo, M. y Charlin, J. (2009). La importancia de las variables morfológicas, métricas y de microdesgaste para evaluar las diferencias en diseños de puntas de proyectil bifaciales pedunculadas: un ejemplo del sur de Patagonia continental. Magallania, 37(1), 99-112.

Franco, N., Gómez Otero, J., Guraieb, G., Goye, S., Cirigliano, N. y Banegas, A. (2010). Variaciones espaciales en diseños de puntas pedunculadas medianas en Patagonia argentina: una nueva aproximación. En J. Bárcena y H. Chiavazza (eds.), Arqueología Argentina en el Bicentenario de la Revolución de Mayo (pp. 281-286). Universidad de Cuyo, ICHSA CONICET.

Gómez Otero, J. (1986-1987). Investigaciones arqueológicas en el alero Potrok Aike (provincia de Santa Cruz). Una revisión de los Períodos IV y $\mathrm{V}$ de Bird. Relaciones de la Sociedad Argentina de Antropología, XVII(1), 173-200.

Goñi, R. A., Espinosa, S., Belardi, J. B., Molinari, R., Savanti, F., Aragone...Rindel, D. (2005). Poblamiento de la estepa patagónica: cuenca de los lagos Cardiel y Strobel. En E. Berberián (ed.), XIII Congreso Nacional de Arqueología Argentina (pp. 7-18). Brujas.

Hocsman, S. y Escola, P. (2006-2007). Inversión de trabajo y diseño en contextos líticos agro-pastoriles (Antofagasta de la Sierra, Catamarca). Cuadernos del Instituto Nacional de Antropología, 21, 75-90.

Iriarte, J. (1995). Afinando la puntería: tamaño, forma y rejuvenecimiento en las puntas de proyectil pedunculadas del Uruguay. Actas del VIII Congreso Nacional de Arqueología Uruguaya (pp. 142-151). SURCOS.

Kuhn, S. (1995). Mousterian Lithic Technology. An Archaelogical Perspective. Princeton University Press.

Loendorf, Ch., Oliver, T., Tiedens, S., Plumlee, R., Woodson, M. y Simon, L. (2015). Flaked-stone projectile point serration: A controlled experimental study of blade margin design. Journal of Archaeological Science: Reports, 3, 437-443. 10.1016/j. jasrep.2015.07.002

Massone, M. (1979). Panorama etnohistórico y arqueológico de la ocupación Tehuelche y Prototehuelche en la costa del Estrecho de Magallanes. Anales del Instituto de la Patagonia, Serie Ciencias Humanas, 10, 69-107.

Massone, M. (1989-1990). Investigaciones arqueológicas en la Laguna Thomas Gould. Anales del Instituto de la Patagonia, Serie Ciencias Humanas, 19, 87-99.

Matera, S., López, L., Pallo, M. C. y Cirigliano, N. (2019). Estudios de impacto e investigación arqueológica: una experiencia complementaria en Tapi Aike (sur de la Provincia de Santa Cruz, Argentina). Práctica Arqueológica, 2(2), 35-51.

Nami, H. (1984). Acerca del uso de retocadores de madera en Patagonia Meridional. Anales del Instituto de la Patagonia, Serie Ciencias Sociales, 15, 51-57.

Nami, H. (1986). Experimentos para el estudio de la tecnología bifacial de las ocupaciones tardías en el extremo sur de la Patagonia continental. Informes de Investigación PREP, 5, 34-45.

Nami, H. (1999). Arqueología en la localidad arqueológica de Pali Aike, cuenca del río Chico (Provincia de Santa Cruz, Argentina). Praehistoria, 3, 189-201.

Nelson, M. (1991). The study of technological organization. Archaeological Method and Theory, 3, 57-100.

Ratto, N. (1991). Análisis funcional de las puntas de proyectil líticas de sitios del sudeste de la Isla Grande de Tierra del Fuego. Arqueología, 1, 151-178.

Ratto, N. (1994). Funcionalidad vs. adscripción cultural: cabezales líticos de la margen norte del Estrecho de Magallanes. En J. L. Lanata y L. A. Borrero (comps.), Arqueología de Cazadores-Recolectores. Límites, Casos y Aperturas. Arqueología Contemporánea, 5, 105-120.

Ratto, N. (2003). Estrategias de caza y propiedades del registro arqueológico en la Puna de Chaschuil (Dpto. Tinogasta, Catamarca, Argentina) [tesis doctoral. Universidad de Buenos Aires].

Smallwood, A. M., Smith, H., Pevny, Ch., y Jennings, T. (2018). The Convergent Evolution of Serrated Points on the Southern Plains-Woodland Border of Central North America. En M. J. O’Brien, B. Buchanan y M. Eren (eds.), Convergent Evolution in Stone-Tool Technology (pp. 203-227). Vienna Series in Theoretical Biology, The MIT Press.

Weitzel, C. (2011). Rotura intencional de artefactos líticos formatizados en la Región Pampeana Bonaerense. Revista del Museo de Antropología, 4, 47-64. http:// publicaciones.ffyh.unc.edu.ar/index.php/antropologia 
